ИЗВЕСТИЯ АКАДЕМИИ НАУК ЭСТОНСКОИ ССР. ФИЗИКА * МАТЕМАТИКА PROCEEDINGS OF THE ACADEMY OF SCIENCES OF THE ESTONIAN SSR.

\title{
ПРИМЕНЕНИЕ ОПТОГАЛЬВАНИЧЕСКОГО ЭФФЕКТА ДЛЯ КАЛИБРОВКИ ДЛИНЫ ВОЛНЫ В ТОНКОСТРУКТУРНОЙ СПЕКТРОСКОПИИ
}

\author{
(Представил К. К. Ребане)
}

\section{- Введение}

В связи с быстрым развитием лазерной техники расширились возможности применения оптогальванического эффекта (ОГЭ) в атомной и молекулярной спектроскопии. Несмотря на то что явление ОГЭ - изменение тока в газовом разряде низкого давления при поглощении монохроматического света - было обнаружено еще в двадцатые годы $\left[{ }^{1}\right]$, в последующие 50 лет ему уделяли мало внимания. Лишь в 1976 г. в связи с аналитическим применением ОГЭ, которое стало возможным благодаря использованию перестраиваемых лазеров на красителях, а также с возможностью стабилизации частоты генерации лазера с помощью этого метода $\left[{ }^{2}\right]$ он попал в центр внимания. Затем $\left[{ }^{3-5}\right]$ ОГЭ на атомных переходах был применен для калибровки лазеров по длинам волн. В последнее время круг объектов исследования оптогальванической спектроскопии расширился на молекулы и молекулярные радикалы в различных разрядах [ $\left.{ }^{6}\right]$. Следует также отметить наблюдение ОГЭ в

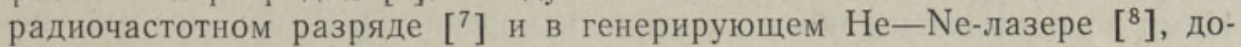
стижение субдоплеровского разрешения в оптогальванической спектроскопии $\left[{ }^{9}\right]$, а также развитие ее в прикладном аспекте $\left[{ }^{10}\right]$.

Точное определение длины волны $\lambda$ наблюдаемых линий, в частности в спектроскопии твердого тела, представляет собой сложную проблему из-за регулярных и случайных отклонений шкал спектральных приборов от истинных значений $\lambda$.

В случае спектров излучения проблема точной калибровки $\lambda$-шкалы решается путем одновременной записи атомных эмиссионных линий от • спектральных ламп. Эти реперные линии можно также использовать для обеспечения точного наложения спектра при многократном накоплении $\left[{ }^{11}\right]$. В случае же спектров возбуждения проблема калибровки оставалась до недавнего времени нерешенной. В частности, шкалу перестраиваемого лазера на красителе трудно сделать хорошо воспроизводимой. Поэтому много внимания было уделено конструированию специальных интерференционных измерителей длины волны $\left[{ }^{12}\right]$, которые, однако, являются дорогостоящими и требуют тщательной юстировки. По этой причине параллельно шли поиски с целью применения ОГЭ, который в ряде случаев может предоставить выгодную альтернативу для калибровки длин волн лазера. Основными преимуществами этого способа являются получение реперов непосредственно в виде электрического сигнала, а также несложность осуществления процесса измерения.

В данной работе описана спектрометрическая система на базе лазера на красителе постоянного действия, позволяющая записать в спектрах 


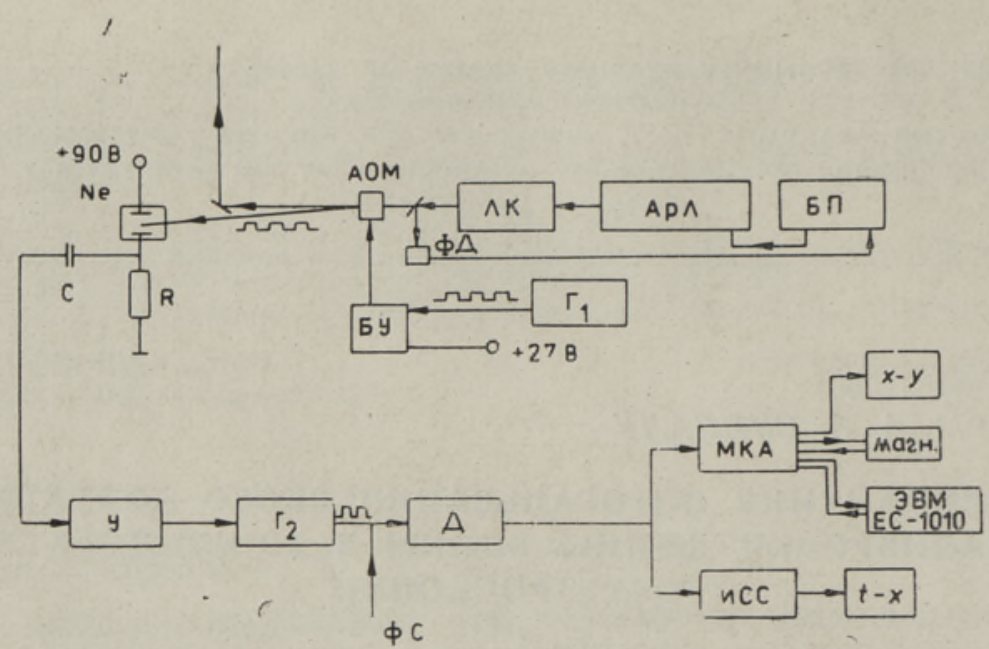

Рис. 1. Схема установки для записи реперных линий ОГЭ: АрЛ аргоновый лазер CR-3, ЛК - лазер на красителе CR-490, БП блок питания, ФД - фотодиод, AOM - акусто-оптический модулятор Mod. 308 с блоком управления БУ, Г 1 - генератор импульсов Г5-54, Ne - лампа ИНС-1, У - микровольтметр В6-4, $\Gamma_{2}$ - генератор импульсов Г5-15, Д - дискриминатор, МКА - многоканальный анализатор, ИСС - измеритель скорости света, $t-x-$ самописец КСП-4, ФС - фотосигнал от ФЭУ, $x-y-$ графопостроитель H-306.

возбуждения люминесценции, регистрируемых счетчиком фотонов, peперные линии ОГЭ неона в виде одинаковых и регулируемых по высоте маркеров. Приводятся результаты практического применения такой системы в лазерной спектроскопии примесных молекул.

\section{Описание системы и результаты измерения}

Нами использовалась обычная схема измерения ОГЭ: переменный сигнал снимался с балластного сопротивления неоновой лампы через конденсатор (рис. 1). В отличие от большинства предыдущих работ, в которых лазерный луч для получения переменного компонента модулировался механически вращающимся диском $\left[{ }^{2-5}\right]$, нами применялся акустооптический модулятор, позволяющий в принципе получить частоту модуляции до 10 МГц (см. также $\left.\left[{ }^{8}\right]\right)$. Кроме того, неотклоненную часть световой волны можно было непосредственно использовать для возбуждения люминесценции объекта. Выходная мощность лазера на красителе (CR-490) поддерживалась постоянной в пределах полосы генерации с помощью системы обратной связи, что весьма целесообразно при измерении спектров возбуждения $\left[{ }^{13}\right]$. Сканирование осуществлялось с помощью трехэлементного двулучепреломляющего фильтра.

В качестве активного элемента, в котором возникал ОГЭ, мы использовали неоновую сигнальную лампочку ИНС-1 с напряжением питания 90 В. При выходной мощности лазера около 10 мВт величина оптогальванического сигнала составляла от 0,1 до 2 В для разных линий $\mathrm{Ne}$, причем знак и временной ход сигналов при прямоугольной модуляции соответствовали таковым, наблюдаемым в лампе с полым катодом в режиме слабого тока $\left[{ }^{14}\right]$.

Для того чтобы регистрировать сигнал ОГЭ с помощью системы счета фотонов одновременно с измеряемым спектром, первоначальный сигнал усиливался по мощности узкополосным усилителем на частоте модуляции, после чего синусоидальным выходным напряжением (при 
повышении установленного порогового значения $\approx 1$ В) запускался генератор $\Gamma_{2}$. В итоге на выходе генератора получали последовательность стандартных импульсов с амплитудой 5 В и с частотой, задаваемой модулирующим генератором $\Gamma_{1}$. Этот сигнал вместе с усиленным сигналом от ФЭУ подавался на вход дискриминатора, а с его выхода - параллельно в многоканальный анализатор и измеритель скорости счета, соединенные с соответствующими периферийными устройствами.

Такая схема позволяет при сканировании лазера получать в измеряемом спектре реперные линии неона с амплитудой, по числу импульсов в секунду равной частоте модуляции, лазерного луча. Поскольку последняя задается генератором $\Gamma_{1}$, то амплитуду реперных линий можно легко установить в удобном для их опознования диапазоне. Так, при измерении спектров возбуждения довольно интенсивной флуоресценции порфиринов сигнал от ФЭУ был в пределах $5 \cdot 10^{4}$ имп/с, и частоту модуляции выбирали при 8,5 кГц. Пример такого спектра вместе с реперными линиями показан на рис. 2. Видно, что линии ОГЭ неона хорошо выделяются на фоне измеряемого спектра. Линии $\mathrm{Ne}$, которые дают одноквантовый ОГӘ в видимой области спектра, т. е. налагаются

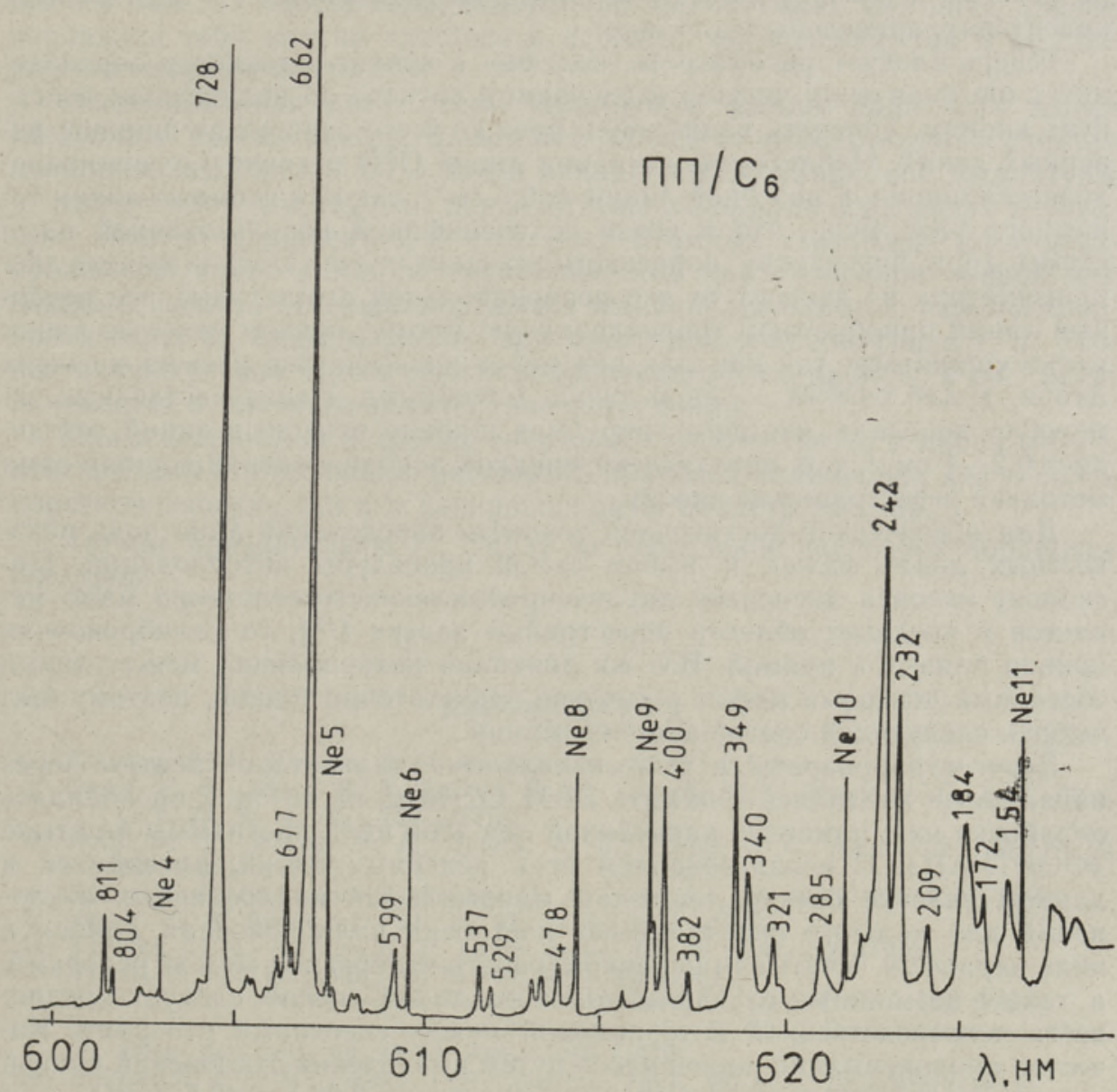

Рнс. 2. Спектр возбуждения флуоресценции протопорфирина IX (ПП) в $н$-гексане $\left(\mathrm{C}_{6}\right)$ при $T=5$ К вместе с реперными линиями ОГЭ неона. Около вибронных линий указаны колебательные частоты $\left(\mathrm{cm}^{-1}\right)$, вычнсленные относительно регистрируемой 0,0 -линии 632,34 нм. Номера реперных линнй $\mathrm{Ne}$ взяты из таблицы. Самая слабая линия № 7 оставалась ниже порога дискриминации. Калибровочная кривая: $\lambda(i)=$ $=7,6 \cdot 10^{-7} i^{2}-3,073 \cdot 10^{-2} i+623,67, i-$ номер канала $(i \leqslant 800)$. 
(Указан знак изменения импеданса лампы в режиме слабого тока; положительный знак соответствует переходам с метастабильных нижних уровней ${ }^{3} P_{0}$ и $\left.{ }^{3} P_{2}\right)$
1) $588,190+$
2) $594,483+$
3) $597,553+$
4) 603,000
5) 607,434
6) 609,616 -
7) $612,845-$
8) $614,306+$
9) $616,359+$
10) $621,728+$
11) $626,650+$
12) $630,479-$
13) $633,444+$

$\begin{array}{ll}\text { 14) } & 638,299- \\ \text { 15) } & 640,225+ \\ \text { 16) } & 650,653- \\ \text { 17) } & 653,288+ \\ \text { 18) } & 659,895- \\ \text { 19) } & 667,828- \\ \text { 20) } & 671,704- \\ \text { 21) } & 692,947- \\ \text { 22) } & 703,241+ \\ \text { 23) } & 717,394- \\ \text { 24) } & 724,517- \\ \text { 25) } & 743,890-\end{array}$

на участок, в котором работают лазеры на родаминовых и оксазиновых красителях, приведены в таблице.

Теперь следует рассмотреть, конечно, и вопрос о точности определения длин волн (или частот) в изучаемом спектре по полученным реперным линиям. Точность калибровки прежде всего зависит от ширины реперных линий. Наблюдаемая ширина линий ОГЭ в данном случае определяется шириной лазерной линии $\approx 0,5 \mathrm{~cm}^{-1}$, так как атомные линии $\mathrm{Ne}$ намного уже. Ясно, что в связи со спецификой использованной нами схемы (при повышении порогового значения сигнал ОГӘ на выходе дйскриминатора не зависит от его первоначальной амплитуды) все реперные линии приобретают трапециевидную форму, причем сильные линии могут уширяться, так как для них порог превышается уже на крыльях линии, а для слабых - лишь около максимума. Непосредственные измерения показали, что регистрируемая ширина реперных линий составляет $0,2-1 \mathrm{~cm}^{-1}$, т. е. практически никакой дополнительной ошибки сама методика регистрации не вносит.

Для обеспечения достаточной точности определения длин волн неизвестных линий важен и выбор самой процедуры интерполяции. Поскольку угловая дисперсия двулучепреломляющего селектора мало меняется в пределах области перестройки лазера $\left[{ }^{15}\right]$, то калибровочная кривая близка к прямой. Все-же линейная интерполяция между двумя соседними реперами может оказаться недостаточно точной, поэтому был выбран следующий способ аппроксимации.

Зарегистрированные в многоканальном анализаторе спектры через канал связи вводились в память ЭВМ ЕС-1010, на которой проводилась обработка их с помощью написанной для этой цели программы на языке «ФОРТРАН». С использованием всех реперных линий, имеющихся в данном участке спектра, методом минимальных квадратичных отклонений для каждого спектра была построена калибровочная кривая в виде параболы. Учитывая неравномерность интервалов между реперами, а также возможные случайные ошибки, такой способ следует предпочесть непосредственной интерполяции между соседними реперами. Качество аппроксимации проверялось путем вычисления отклонений кривой от реперных точек - они составляли не более $0,02 \mathrm{нм}\left(0,5 \mathrm{~cm}^{-1}\right)$.

Искомые длины волн линий исследуемого спектра вычислялись на основе этой калибровочной кривой, причем все линии маркировались на графическом дисплее по положению максимума. Кроме того, программа включала вычисление колебательных частот (в $\left.\mathrm{cm}^{-1}\right)$ по расстоянию до частоты регистрации, калиброванной по эмиссионным линиям неона. 
В итоге частоты вибронных линий определялись с точностью $1 \mathrm{~cm}^{-1}$, что вполне достаточно, поскольку ширина линий в типичных спектрах Шпольского составляет несколько обратных сантиметров, а в случае большого неоднородного уширения и селективного возбуждения $5-10 \mathrm{~cm}^{-1}$.

В коротковолновой части полосы генерации родамина $6 Ж$, где отсутствуют линии ОГЭ неона (от 570 до 588 нм), мы пользовались теми же калибровочными кривыми, построенными по более длинноволновым реперным линиям. Правомерность использования такой экстраполяции проверялась как путем непосредственной калибровки монохроматором, так и имитацией этой процедуры в длинноволновой части, опуская самые коротковолновые линии ОГЭ при аппроксимации и экстраполируя их положения на основе полученной по остальным линиям аппроксимационной кривой. Точность определения осталась практически такой же, какой она была между реперными линиями, лишь на самом краю полосы генерации отмечена возможность увеличения ошибки до $5 \mathrm{~cm}^{-1}$.

Описанным выше способом был обработан целый ряд квазилинейчатых спектров возбуждения разных порфиринов в $н$-алкановых матрицах, причем каждый спектр содержал 20-30 линий. Такая методика вполне оправдала себя как по простоте и удобству применения, так и по скорости и точности обработки.

Использованная нами схема калибровки шкалы длин волн имеет следующие преимущества. Во-первых, запись непосредственно в спектре калибровочных линий ОГЭ почти одинаковой регулируемой амплитуды псзвсляет удсбным образом привязать значения длин волн линий изучаемого спектра к. значениям $\lambda$ известных атомных переходов. Во-вторых, проведение аппроксимации параболической калибровочной кривой для каждого спектра отдельно исключает ошибки, связанные с переюстировкой лазера, со сдвигом шкалы при изменении температуры и т. п. Точность определения частот из откалиброванного спектра $\delta v \leqslant 1 \mathrm{~cm}^{-1}$ ограничивается в основном шириной лазерной линии.

Применение ОГЭ в других инертных газах, а также в парах металлов позволяет, в принципе, расширить диапазон калибровки как в более коротковолновую, так и в длинноволновую область спектра.

Авторы признательны П. Сарву за проведение численной обработки спектров.

\section{Л И Т Е Р А Т У Р А}

1. Penning, F. M. Physica, 8, № 4, 137-140 (1928).

2. Green, R. B., Keller, R. A., Luther, G. G., Schenk, P. K., Travis, J. C. Appl. Phys. Lett., 29, № 11, 727-729 (1976)

3. King, D. S., Schenk, P. K., Smyth, K. C., Travis, J. C. Appl. Opt., 16, № 10, 2617-2619 (1977).

4. Keller, R. A., Engleman, R., Palmer, B. A. Appl. Opt., 19, № 6, 836-837 (1980).

5. Nestor, J. R. Appl. Opt., 21, № 22, 4154-4157 (1982).

6. Webster, C. R., Rettner, C. T. Laser Focus, 19, № 2, 41-52 (1983).

7. Suzuki, T. Opt. Commun., 38, № 516, 364-368 (1981).

8. Кукк П. Л., Фрейбере А. М. Квант. электроннка, 11, вып. 3, 457-462 (1984).

9. Lawler, J. E., Ferguson, A. I., Goldsmith, J. E., Jackson, D. J., Schawlow, A. L. Phys. Rev. Lett., 42, № 16, 1046-1049 (1979).

10. Goldsmith, J. E. M., Lawler, J. E. Contemp. Phys., 22, № 2, 235-248 (1981).

11. Саари П. М. Автореф. дис. д-ра физ.-мат. наук. Тарту, 1980.

12. Product Summary. New York, Burleigh Instruments Inc., 1981.

13. Rebune, K. K., Avarmaa, R. A. Chem. Phys., 68, № 1/2, $191-200$ (1982).

14. Smyth, K. C., Schenk, P. K. Chem. Phys, Lett, 55, № 3, 466-472 (197S).

15. Қачанов А. А. Қвант. электроника, 9, вып. 7, 1458-1462 (1982).

Институт физики

Акдемии наук Эстонской ССР
Поступила в редакцию 24/VI 1983 


\section{OPTOGALVAANILISE EFEKTI KASUTAMINE LAINEPIKKUSE KALIBREERIMISEKS PEENSTRUKTUURSPEKTROSKOOPIAS}

On kirjeldatud süsteemi, mis võimaldab footonloendiga mõōtmisel registreerida luminestsentsi ergastusspektreis neooni reeperjooni kõrguse järgi normeeritud markeritena. Akustooptiliselt moduleeritud pidevvärvlaseri skaneerimisel saadakse neoonlambist optogalvaaniline signaal, kusjuures vahelduvsignaal muudetakse standardimpulsside jadaks, nii et fotoloendiga mõõdetav impulsside arv on määratud modulatsioonisagedusega. Tingimusel, et on valitud sobiv diskriminatsiooninivoo, annab selline moodus uuritava spektri føonil peaaegu ühesuguse amplituudiga optogalvaanilised reeperjooned. Paljukanalilises analüsaatoris salvestatud spektreid on töödeldud miniarvutiga ja leitud reeperjoonte abil lähenduskõverad lainepikkuste kalibreerimiseks. Kirjeldatud süsteemi on kasutatud vibroonjoonte sageduste määramiseks porfüriinide Spolski spektri tüüpi ergastusspektritest ja see kindlustas täpsuse $1 \mathrm{~cm}^{-1}$.

\section{R. AVARMAA, A. SUISALU}

\section{APPLICATION OF OPTOGALVANIC EFFECT FOR WAVELENGTH CALIBRATION IN FINE LINE SPECTROSCOPY}

A system is described that enables to record in the fluorescence excitation spectra marker lines of neon discharge, using a photon counter. The output beam of a tunable dye laser is modulated by an acoustooptical modulator, and optogalvanic signals are obtained from a small neon lamp. The nonuniform ac signal is converted into squarewave pulses of standard height, whereas the photon counting system detects the number of pulses equal to the modulation frequency. In case of a suitable discrimination level, one obtains optogalvanic marker lines of almost equal intensity superimposed on the excitation spectra studied. Digitally recorded spectra are transferred into a minicomputer, and a least squares' approximation for calibration curve through the marker lines is found for each spectrum. Using this system, frequencies of vibronic lines in the excitation spectra of porphyrin molecules in $\mathrm{n}$-alkane matrices are determined with $1 \mathrm{~cm}^{-1}$ accuracy. 